\title{
Género y matemáticas. Colombianas pioneras en el campo de las matemáticas
}

\author{
Clara Helena Sánchez B. \\ Departamento de Matemáticas, Universidad Nacional de Colombia \\ Artículo de posesión para el ingreso como miembro correspondiente a la \\ Academia Colombiana de Ciencias Exactas, Físicas y Naturales el 14 de junio de 2017
}

\begin{abstract}
Resumen
En este trabajo me propongo destacar a las primeras colombianas que obtuvieron un doctorado en matemáticas; para ello es relevante tener en cuenta el contexto nacional e internacional, pues revela la importancia de ese hecho. Una breve reflexión sobre las matemáticas y el género permite hallar respuestas sobre la baja representación femenina en la comunidad matemática en Colombia y en el mundo, y sobre la invisibilidad de las mujeres en este campo. (C) 2017. Acad. Colomb. Cienc. Ex. Fis. Nat.
\end{abstract}

Palabras claves: Matemáticas; Género; Colombia; Historia de las matemáticas.

Gender and mathematics. Colombian pioneering women in mathematics

\begin{abstract}
In this paper, I intend to enhance the place of the first Colombian women who obtained a PhD degree in Mathematics contextualizing its importance both in the national and the international contexts. A brief discussion on the relation between gender and Mathematics allows to find answers regarding the low representation of women in the mathematical community and their invisibility in this field. (C) 2017. Acad. Colomb. Cienc. Ex. Fis. Nat.
\end{abstract}

Key words: Mathematics; Gender; Colombia; History of Mathematics.

\section{Introducción}

Recibo la distinción de haber sido aceptada como Miembro Correspondiente de la Academia Colombiana de Ciencias Exactas, Físicas y Naturales, conjuntamente con Tatiana Toro, como un reconocimiento a todas las matemáticas que nos hemos formado en el país desde la década de 1960. Somos las primeras matemáticas en ser aceptadas como miembros correspondientes en los 80 años de la Academia. La primera mujer en ser recibida fue la recientemente fallecida botánica María Teresa Murillo, en 1975; desde entonces, varias biólogas, químicas y físicas han ingresado a la institución. Sin embargo, la representación femenina sigue siendo baja, de 208 miembros, apenas 34 son mujeres, dos de ellas miembros honorarias, nueve, de número y 23 , miembros correspondientes, incluidas las académicas que están por posesionarse.

Mi interés en las matemáticas y su relación con el género se suscitó de manera circunstancial: comenzó cuando traduje un artículo sobre mujeres pioneras en matemáticas (Sánchez, 1988) que me pareció interesante. En 2003 fui invitada a participar en la Cátedra Manuel Ancizar sobre ciencia y tecnología en Colombia, en una sesión sobre ciencia y género en la que intervine al lado de Florence Thomas, reconocida por su liderazgo en los estudios de género en
Colombia y Coordinadora del Grupo Mujer y Sociedad, y de Patricia Tovar, antropóloga que en la época hacía una investigación sobre este tema auspiciada por la Organización de Estados Iberoamericanos (OEI) y la Unesco. Consideré esa invitación como un reto que debía afrontar y dediqué mi charla a la representación femenina en la Facultad de Ciencias de la Universidad Nacional y, particularmente, a las mujeres matemáticas, y la titulé "Matemáticas 90-60-90". Una versión mejorada titulada "Mujeres en la Facultad de Ciencias de la Universidad Nacional" fue presentada en el seminario "Las mujeres colombianas en el sistema de ciencia y tecnología: obstáculos y logros” (Sánchez, 2004).

Para aquella conferencia debí estudiar un poco sobre el tema y desde entonces me han invitado a otros eventos para hablar sobre las mujeres en las matemáticas. La profesora Doris Hinestroza me invitó en el 2014 a la celebración de los 60 años de la fundación del Departamento de Matemáticas de la Universidad del Valle y fue entonces cuando pensé que debíamos visibilizar a las matemáticas colombianas, y por ello dediqué la charla a exaltar a las mujeres pioneras

\section{Correspondencia:}

Clara Helena Sánchez B., chsanchezb@unal.edu.co

Recibido: 09 de junio de 2017

Aceptado: 2 de septiembre de 2017 
en el campo de las matemáticas en el país. En el Congreso Colombiano de Matemáticas del 2015, celebrado en Manizales, organicé con otras colegas un foro sobre Las matemáticas colombianas, aprovechando la ambigüedad del título, del cual se hizo un video que puede verse en youtube (https:// www.youtube.com/watch?v=QQh3HG9tNs4). En dicho foro estuvieron presentes cuatro colombianas, Margarita Botero, Myriam Muñoz, Débora Tejada y Doris Hinestroza, primeras colombianas en obtener un doctorado en este campo, cuyas trayectorias inspiran estas palabras, así como mis investigaciones en torno a la historia de las matemáticas en general (Sánchez, 1986, 1995, 1996, 1997, 2007c), de las matemáticas en Colombia (Sánchez, 1999, 2002, 2007a, 2007b, 2009, 2012), y al papel fundamental de la historia como recurso didáctico para la enseñanza de las matemáticas (Sánchez, 2012).

Para comenzar, es importante señalar que un famoso libro sobre la historia de las matemáticas, quizás uno de los primeros, se titula Men of Mathematics (Bell, 1937), y en él el autor hace un recorrido a lo largo del desarrollo de las matemáticas valiéndose de las biografías de unos cuantos matemáticos, desde Zenón (siglo V a.C.) y Euclides (siglo III a.C.) hasta los más importantes de comienzos del siglo $\mathrm{XX}$. En el libro no se menciona a ninguna mujer, a pesar de que en la historia ha habido matemáticas destacadas, así sean muy pocas. Frente a este antecedente, los libros de Osen (1974) y Henrion (1997), ambos titulados Women in Mathematics, están dedicados a resaltar el papel de las mujeres en el desarrollo de las matemáticas. Osen (1974) rescata del olvido a ocho mujeres, desde Hipatia en el siglo IV a.C. hasta Emmy Noether en los comienzos del siglo XX, $\mathrm{y}$ presenta las condiciones sociales en que estas mujeres se desenvolvieron y cómo, a pesar de serles adversas, lograron obtener resultados sobresalientes en este campo. El libro termina con un capítulo en el que la autora expresa su deseo de que se estimule en su medio el estudio de las matemáticas entre las mujeres.

Henrion (1997), por su parte, pone de manifiesto algunos de los prejuicios que existen sobre los matemáticos en general y sobre las matemáticas en particular, y cita el ejemplo de mujeres activas en esta disciplina en los Estados Unidos para refutar esos prejuicios, entre los cuales quiero mencionar los que paso a detallar.

El primero se finca en la afirmación de que los matemáticos trabajan en completo aislamiento, lo que es fácil de refutar, ya que sin interlocutores válidos no es posible hacer ciencia y, además, porque se trabaja en grupo con frecuencia, lo cual se evidencia en los múltiples artículos y libros escritos por más de un autor.

El segundo afirma que las matemáticas no son para las mujeres, lo cual queda refutado en este trabajo con los ejemplos de las mujeres dedicadas a su desarrollo.

El tercero plantea que las matemáticas son para hombres jóvenes, mito que, infortunadamente, se ve reiterado con el otorgamiento de la Medalla Fields, creada en 1936 por el matemático canadiense John Charles Fields (1863-1932) y considerada como el Nóbel en matemáticas, la cual se entrega a matemáticos sobresalientes menores de 40 años. Fields dejó un fondo para conceder el premio, el cual es otorgado desde 1950 por la Unión Matemática Internacional cada cuatro años, con una retribución de $10.000 €$. La distinción se entrega con ocasión del Congreso Internacional de Matemáticas a dos o cuatro matemáticos y hasta ahora 54 de ellos han hecho merecedores de la distinción. En el 2014 la Medalla fue otorgada por primera vez a un latinoamericano, el brasilero Artur Avila, y a una mujer, la iraní Maryam Mirzakhani. Esta destacada matemática nació en Teherán en 1977 y desde muy joven demostró una gran capacidad para las matemáticas. En 1994, con 17 años, ganó la medalla de oro en la Olimpiada Internacional de Matemáticas con la máxima puntuación (42 de 42 puntos posibles). Licenciada en Matemáticas de la Universidad de Sharif (Teherán) en 1999, realizó sus estudios de doctorado en la Universidad de Harvard, y como profesora del Departamento de Matemáticas de la Universidad de Stanford se concentró en el estudio de las superficies hiperbólicas, los espacios de móduli y los sistemas dinámicos. Falleció a los 40 años, el 15 de julio pasado, justamente cuando me encontraba revisando este artículo.

Las matemáticas y la política no se mezclan es otro prejuicio que en nuestro medio se han encargado de refutar Antanas Mockus y Sergio Fajardo, alcaldes de Bogotá y Medellín, respectivamente. Fajardo, además, fue gobernador de Antioquia, y ambos han sido candidatos presidenciales.

Solamente las mujeres blancas hacen matemáticas es otra afirmación recientemente desmentida por los hechos recreados en la película Hidden Figures, sobre la historia de tres matemáticas de raza negra que lucharon contra la segregación, la discriminación y el sexismo trabajando en la Nasa durante la década de 1960, en pleno auge de la carrera por la conquista del espacio en Estados Unidos. En Colombia hay mujeres de todas las razas dedicadas a las matemáticas, ya sea como maestras de educación escolar o como matemáticas profesionales.

Otra interesante aseveración es que las matemáticas son totalmente objetivas, aseveración relacionada íntimamente con concepciones filosóficas acerca del conocimiento matemático. La creencia de que la matemática es objetiva, verdadera y libre de influencias sociales es responsable, en buena parte, del prestigio social del que goza. Desde la aparición de las geometrías no euclidianas es claro que la matemática no ofrece verdades absolutas y es por ello que se habla de teoremas, es decir, "verdades" relativas que se circunscriben a una determinada teoría.

A los ya señalados yo añadiría otros prejuicios comunes en nuestro medio: los matemáticos son muy inteligentes, pero locos, despistados, poco sociables, estrictos, y demasiado exigentes cuando de corregir exámenes se trata. Algunos de estos mitos son abordados en el librito Du côté des mathématiciennes (Del lado de las matemáticas, 2000), en el cual sus autoras (Boisseau, et al., 2000) abordan el tema 
de las matemáticas y las mujeres dedicadas a este campo en Francia. En la contra carátula de la publicación se lee: "Aunque la historia les ha dado un ínfimo lugar, ellas son hoy en día numerosas en Francia y en todas partes". Mediante citas y anécdotas, las autoras discurren sobre el tema que hoy nos convoca, aportando datos sobre la contribución de las matemáticas europeas y develando de manera simpática su realidad en ese continente. El libro también resalta el papel de las mujeres en la enseñanza de las matemáticas en todos los niveles de escolaridad, dándoles la importancia que se merecen.

Recientemente, Paycha, et al. (2016) también asumieron la tarea de visibilizar a algunas mujeres matemáticas europeas activas en diferentes especializaciones. Las autoras presentaron una galería de 13 mujeres dedicadas a las matemáticas, pues como afirman en la introducción, "hoy en día las mujeres tienen dificultades para desarrollar una carrera en el mundo académico de las matemáticas; presiones sociales y dudas personales las restringen para hacerlo". La exhibición que montaron se tituló "Mujeres matemáticas a través de Europa. Una galería de retratos”, y se realizó por primera vez en el Congreso Europeo de Matemáticas en julio de 2016 (http://womeninmath.net/venue/bogotacolombia/). Tuvimos la fortuna de albergar la exhibición en la Universidad Nacional del 4 al 21 de abril de 2017 y de organizar un conversatorio sobre el tema. Sorprende que en países del primer mundo las mujeres aún sigan teniendo que sortear obstáculos de todo tipo para desempeñarse como profesionales de las matemáticas.

\section{Matemáticas y género}

Lo primero que hay que decir es que el título bien podría haber sido "Matemáticas y sexo", pero como bien afirma Ernest (1976), el "título puede ocasionar una sonrisa. Para una persona medianamente educada podría ser muy difícil imaginar dos temas tan poco relacionados. Sin embargo, es una de esas cuestiones de nuestra cultura y estructura social que hace que sea muy relevante para tener oportunidades en el mundo de las matemáticas".

Antes de continuar, habría que señalar cuál es la diferencia entre sexo y género. En este sentido, precisamente en este año, la revista National Geographic dedicó un número especial al género titulado "Género. La revolución. El Panorama cambiante del género" (National Geographic, 2017), en el cual se lee lo siguiente: "Liberada del concepto binario de hombre y mujer, la identidad de género presenta un panorama cambiante. ¿Puede la ciencia ayudarnos a encontrar el rumbo?". El desafío está planteado. Si en biología se habla de sexo masculino o femenino, géneros hay varios: masculino, femenino, gay, lesbiana, transexual, bisexual. "Género y sexo no son sinónimos. El género es una construcción sociocultural, una categoría que clasifica y organiza las relaciones sociales entre hombres y mujeres" (Santos, et al., 2006).

En biología algunos autores prefieren la palabra sexo para referirse a distinciones de tipo genital o de cromosomas, en tanto que el género se refiere a las diferencias entre hombres y mujeres que tienen que ver más con las influencias sociales o del entorno. Sin embargo, la biología no puede separarse de la influencia occidental que perpetúa la dualidad entre mente y cuerpo; además, separar la biología de la cultura es muy difícil, pues las dos se influyen mutuamente (Halpern, et al., 2007).

Por otra parte, en los estudios de los psicólogos sobre las habilidades cognitivas de una persona para las matemáticas, se revisan esencialmente tres aspectos: las habilidades espaciales, las verbales y las cuantitativas, y se concluye que los hombres tendrían más habilidades espaciales y cuantitativas, en tanto que las mujeres se destacan en las verbales. En el estudio de Halpern, et al., se señala que hay diferentes tipos de matemáticos y que en dicha disciplina las personas se desempeñan independientemente del género que tengan.

Cuando se hace el recuento histórico de las consideraciones sobre las mujeres y su papel es posible entender las dificultades que han tenido para dedicarse a lo que les gusta. Ya Aristóteles pensaba que las mujeres eran seres intelectualmente inferiores cuyo cerebro era más pequeño, por lo cual eran incapaces de hacer ciencia y mucho menos matemáticas, dado su carácter abstracto:

"Las mujeres son defectuosas, débiles, incompletas, menos musculosas, su carne más blanda, sus rodillas más juntas, su voz más débil. El cuerpo femenino, débil e indefenso, tiene un cerebro más pequeño." (Aristóteles, La historia de los animales, 638b, 7-24, citado en Perdomo, s.f.).

Por el contrario, entre los pitagóricos, hombres y mujeres hacían matemáticas. Téano de Crotona, alumna y luego esposa de Pitágoras, participó activamente en los descubrimientos matemáticos como veremos en el siguiente apartado. La pregunta obvia es, ¿por qué hubo ese cambio hacia las mujeres en tan pocos siglos, del V al III a.C.? No tengo respuesta. Lo que sí tengo claro es que la influencia de Aristóteles en la cultura occidental es muy significativa, y su creencia sobre las mujeres de alguna manera se mantiene, reforzada por la de otros eminentes pensadores como Darwin:

"De todas maneras, es probable que el hombre se haga tan superior en dotación mental a la mujer como el pavo real en plumaje ornamental a la pava". (Darwin, La descendencia del hombre y la selección en relación al sexo, 1871, citado por Perdomo, s.f.).

En 1880, con los primeros estudios sobre matemáticas y género, los científicos del momento le dieron la razón a Aristóteles (Clarke, 1887). Las cosas comenzaron a cambiar cuando se desarrollaron métodos más precisos para evaluar las diferencias. Hoy el panorama es bastante diferente.

No sobra recordar que hasta bien entrado el siglo XX, en Colombia las mujeres y los indígenas se consideraban menores de edad, sujetos de tutela, e imposibilitados de tomar decisiones autónomas. Solo hasta 1954 se les otorgó a las mujeres el derecho al voto, el cual ejercieron por primera vez en 1957. La situación cambió radicalmente, por lo menos en la ley, cuando la Corte Constitucional, creada en 1991, revisó el código civil de 1932, derogando la mayoría de sus artículos y estableciendo la equidad de género. 
Según Perdomo (s.f.):

"En las últimas décadas existe un interés renovado en la medición de partes del cerebro y localización de las funciones cognitivas de forma exacta en las distintas regiones del cerebro. El último episodio de la búsqueda de las claves de la diferencia por recurso a lo biológico lo constituye la teoría de la lateralización cerebral".

Dichas teorías plantean que los hemisferios derecho e izquierdo del cerebro procesan la información y controlan funciones y habilidades cognitivas de manera diferente, pero, ¿cómo influye esto en el desarrollo cognitivo de las mujeres? He ahí la cuestión.

Desde hace unos años abundan las investigaciones sobre este tema desde otros ángulos. Uno de ellos se plantea visibilizar a las mujeres de ciencia en la historia y reflexiona sobre el ambiente en el que se desenvolvieron. Otra aproximación analiza desde el punto de vista pedagógico lo que debe hacerse para que las barreras que aún subsisten puedan superarse. Por último, se estudia el tema desde un punto de vista epistemológico en el campo de la historia y la filosofía de la ciencia.

En Colombia apenas ahora se comienza a explorar el tema, y se requiere el apoyo institucional para adelantar investigaciones serias sobre el asunto. Es posible encontrar algunos datos en internet sobre los resultados, por ejemplo, de las pruebas Saber (Rojas \& Correa, 2014) o de las Olimpiadas Colombianas de Matemáticas. Por otra parte, en el 2016 se creó la Red Colombiana de Mujeres Científicas (https://www.facebook.com/MujerCientificaColombia/), cuyo objetivo es "incentivar en las niñas la opción de dedicar su vida profesional a la investigación científica y surge de la preocupación por la falta de más mujeres que se dediquen a la ciencia" (http://www.elespectador.com/noticias/ ciencia/nace-red-colombiana-de-mujeres-cientificasarticulo-625715). El proceso de constitución legal de dicha Red ha contado con el apoyo de la Academia Colombiana de Ciencias Exactas, Físicas y Naturales. Ángela Camacho, física egresada de la Universidad Nacional, $\mathrm{PhD}$ en este campo, docente de la Facultad de Ciencias de la Universidad de Los Andes y miembro de la Academia, es la actual presidenta de la Red, que hoy integra a 39 científicas. Se han realizado numerosas actividades para visibilizar a las científicas colombianas y promover la formación en ciencias de las niñas y jóvenes de nuestro país. Este trabajo pretende, entre otras cosas, aportar un grano de arena en este camino.

\section{Mujeres pioneras en matemáticas}

De manera muy sucinta voy a mencionar a las mujeres matemáticas rescatadas del olvido desde el pasado remoto de la escuela pitagórica hasta comienzos del siglo XX (Sánchez,1988). En internet hoy es posible encontrar muchas páginas dedicadas a ellas, y cada día surgen nuevos nombres mencionados en los artículos y libros sobre el tema, como puede verse en la bibliografía que cito. A continuación hago un breve recuento de algunas de las mujeres que han dejado su impronta en el campo de las matemáticas.
Como ya se mencionó, Téano de Crotona (siglo V a.C.) fue una de las primeras filósofas y matemáticas de la historia. Al parecer escribió varios tratados de filosofía, matemáticas y medicina y a ella se le atribuye el teorema matemático de la "proporción áurea". Defendió, asimismo, el papel de la mujer como esposa y madre http:/www.mujeresenlahistoria. com/2013/02/la-pitagorica-teano-de-crotona-siglo-vi.html).

Hipatia, hija del matemático y astrónomo Teón, nació en Alejandría (370-415). Se cree que ayudó a su padre a escribir los comentarios al Almagesto de Tolomeo, a los Elementos de Euclides y a las Cónicas de Apolonio, pero sus trabajos se encuentran perdidos. Dedicó su vida a la filosofía, las matemáticas y la astronomía, y fue asesinada por cristianos fanáticos que la consideraban satánica.

La francesa Gabrielle Emilie de Breteuil, Marquesa de Chatelet (1706-1749), fue hija de un funcionario francés rico y poderoso, quien, reconociendo las capacidades intelectuales de su hija, la educó en varias lenguas y en matemáticas, campo al cual se dedicó desde los 27 años. Fue amante de Voltaire, quien la convirtió en una ferviente anglófila, por lo cual se dedicó al estudio de la obra de Newton y tradujo al francés sus Principia, con extensos y valiosos comentarios y adiciones redactados con la ayuda del físico Alexis Clairaut (1713-1765). También estudió cuidadosamente a Leibniz y de él publicó Las instituciones de la física.

La italiana Maria Gaetana Agnesi (1718-1799), hija de un profesor de matemáticas de la Universidad de Bolonia, quien la educó desde pequeña, manejaba varios idiomas y fue una gran defensora de la educación superior para la mujer. Estudió las matemáticas de Descartes, Fermat, Newton, Leibniz, Euler y los Bernoulli, lo cual le permitió escribir un texto para la enseñanza del cálculo diferencial e integral titulado "Instituciones analíticas para el uso de la juventud italiana", publicado en 1748 en dos volúmenes. Este fue el primer texto para la enseñanza del cálculo diferencial e integral. A pesar de haber recibido el reconocimiento de la Academia de Ciencias de París en 1749, irónicamente es recordada por una curva cúbica llamada La bruja de Agnessi (http://commons.wikimedia.org/wiki/File:Bruja_de_Agnesi. gif\#mediaviewer/File:Bruja_de_Agnesi.gif). La Academia de Ciencias de Bolonia la eligió como unos de sus miembros.

La matemática francesa Sophie Germain (1776-1831) estudió matemáticas a escondidas de sus padres y publicó artículos sobre sus análisis bajo el seudónimo de M. Leblanc. Sostuvo comunicación tanto con Lagrange como con Gauss quienes, al descubrir quién era su interlocutora, le expresaron su admiración y solicitaron para ella un doctorado Honoris causa que no alcanzó a recibir, pues murió tempranamente de cáncer. Hizo aportes significativos a la teoría de números y la teoría de la elasticidad.

Augusta Ada Byron, Condesa de Lovelace, fue la hija (1815-1852) de Lord Byron y Anna Isabella Milbanke, una rica heredera inglesa educada en matemáticas y astronomía que se preocupó por la educación de su hija en estos temas. 
Entre sus tutores se contaron Charles Babbage (1791-1871), precursor de los computadores, y el ingeniero italiano Luigi Federico, conde de Menabrea, quien publicó un trabajo sobre las máquinas de Babbage, el cual fue mejorado sustancialmente por Lady Lovelace en 1843. Babbage y Ada quedaron relegados al olvido casi completo hasta que las computadoras fueron reinventadas después de la Segunda Guerra Mundial. En 1961, el Barón Bertram V. Bowden, pionero de la computación en Inglaterra, reeditó los escritos de 1843 y estos fueron publicados en forma de libro por Dover con el título de "Charles Babbage and his calculating machines", trabajo en el cual la Condesa de Lovelace fue reconocida un siglo después como pionera de las máquinas calculadoras.

La rusa Sonya Kovalevska (1850-1891) fue la primera matemática de importancia en su país y la primera mujer que consiguió una plaza de profesora universitaria en Europa. Nació y se crio en el seno de una familia gitana rusa de buena formación académica. Entre sus trabajos figuran los siguientes: "Sobre la teoría de las ecuaciones diferenciales", que aparece en el Journal de Crelle, y "Sobre la rotación de un cuerpo sólido alrededor de un punto fijo", por el cual obtuvo un importante premio otorgado por la Academia de Ciencias de París en 1888. Sin embargo, en Rusia no fue aceptada para estudiar en la universidad y, como no era bien visto que las mujeres viajaran solas, se casó para poder viajar a Alemania donde fue alumna de Weierstrass.

La alemana Emmy Noether (1882-1935) estudió matemáticas en la Universidad de Erlangen-Núremberg, donde su padre impartía clases. Fue invitada a formar parte del Departamento de Matemáticas de la Universidad de Gotinga, pero a ello se opuso el Departamento de Filosofía. Sin embargo, ejerció como Privatdocent impartiendo los cursos de David Hilbert (1861-1943), quien le ofreció todo su apoyo. Es conocida por sus contribuciones de fundamental importancia en los campos de la física teórica y el álgebra abstracta. Hilbert y Einstein, entre otros, la consideraron la mujer más importante en la historia de las matemáticas. Revolucionó las teorías de anillos, cuerpos y álgebras, y es autora del teorema de Noether, el cual explica la conexión fundamental entre la simetría en física y las leyes de conservación. En su libro, Byers \& Williams (2010) le hacen un especial reconocimiento.

\section{Prejuicios contra las matemáticas}

Aunque es difícil encontrar un ser humano que no haya tenido contacto con las matemáticas, y se sabe que es una disciplina necesaria en la mayoría de los campos científicos y técnicos, hay mucho desconocimiento acerca de qué son las matemáticas y qué hacen los que se dedican a su estudio. El reconocido matemático del grupo Bourbaki, Jean Dieudonné (1906-1992) afirma que "algunos científicos destacados en otros campos sólo cuentan a menudo con nociones aberrantes acerca del trabajo de los matemáticos." (Dieudonné, 1989), lo cual ratifica Ernest (2000):
"Generalmente, las matemáticas son consideradas como algo difícil, frío, abstracto, teórico y que se escapa a la razón, pero a la vez como algo importante y característico del género masculino. Además, se las considera remotas e inaccesibles para todos aquellos que no sean muy inteligentes o que no tengan la mente preparada para las matemáticas. En el Reino Unido hay muchas personas que en su vida profesional utilizan a diario matemáticas a un alto nivel de competencia para realizar cálculos, hacer gráficos y para trabajar con su ordenador y aun así siguen diciendo que no se les dan las matemáticas. En comparación con la desgracia de ser analfabeto, el 'anumerismo' es exhibido en muchos casos con orgullo entre las personas cultas de los países anglosajones occidentales."

Para Dieudonné, "un matemático es alguien que ha publicado al menos la demostración de un teorema no trivial". Al respecto habría que decir que lo que antes se hacía por talento y por consagración a la disciplina, hoy son características que debe tener quien opta a un doctorado y va a trabajar en una institución universitaria de buen nivel donde se haga investigación en matemáticas. Pero son muchos los matemáticos de profesión que, así no hayan demostrado teoremas originales, han aportado al desarrollo de la disciplina desde otros escenarios como la docencia, la publicación de textos, o apoyando el avance de las matemáticas en instituciones o asociaciones, por lo cual todos ellos deben obtener reconocimiento.

En 1886, la Universidad de Columbia otorgó el primer grado de $\mathrm{PhD}$ en matemáticas a la matemática norteamericana Winifred Edgerton. En los siguientes 40 años, obtuvieron ese mismo grado otras 99 mujeres, entre ellas, Christine LaddFranklin, quien recibió su grado en 1926 después de haber sido rechazada anteriormente por la Universidad Johns Hopkins por el hecho de ser mujer (http://americanhistory.si.edu/ blog/2013/03/finding-female-pioneers-in-mathematics.html).

El trabajo de Henrion (1997), como ya anoté, está dedicado a explorar las dificultades que una mujer de nuestro tiempo puede tener para desempeñarse como profesional de las matemáticas en sus más altos niveles. A través de las entrevistas que sostuvo con diez destacadas matemáticas norteamericanas Henrion logra refutar los mitos más populares acerca de las matemáticas y de quienes nos dedicamos a ella. Así que la reflexión sobre la baja participación femenina en los niveles superiores de la disciplina matemática tiene este ingrediente adicional que la diferencia de la reflexión general sobre ciencia y género, o sobre física y género, o química y género, es decir, los prejuicios en torno a las personas que se dedican a las matemáticas que ya mencioné.

El problema de las matemáticas y el género ocupa un lugar particular en las reflexiones sobre ciencia y género. Una simple búsqueda en internet refleja la inquietud generalizada en Estados Unidos, Canadá y Europa sobre la baja participación de las mujeres en las carreras de ciencias, aunque en áreas como la biología o la química la brecha entre la participación de hombres y mujeres no es tan 
acentuada. Una manera de afrontar el problema ha sido la creación de asociaciones de mujeres matemáticas, físicas, químicas, o biólogas, enntre las cuales cabe mencionar la Women in Biology, la Association for Women in Science - AWIS, la Association for Women Geoscientists - AWG, la National Association of Women Pharmacists - NAWP, el Women Chemists Group, la Association for Women in Mathematics - AWM, la American Association of Physics Teachers, la Women in Physics - WIP, el ASA Committee on Women in Statistics, la Com Ciência - Mulheres na Ciência, y la Women in Astronomy - CSWA. La queja generalizada en estas asociaciones es que la representación femenina es muy baja, y uno de sus objetivos es estimular el estudio de la ciencia entre las mujeres. Por otro lado, se afirma que no hay equidad en cuanto a la remuneración salarial, pues la de las mujeres está por debajo de la de los hombres en los casos en que logran posiciones en universidades o en la industria. La situación es similar en las asociaciones de Canadá, Gran Bretaña y la Unión Europea, en donde se han hecho varios estudios sobre los niveles de participación femenina que expresan la preocupación por los bajos niveles de participación femenina y mencionan las acciones que se están desarrollando para mejorarlos. En este contexto es válida la pregunta sobre cómo son las cosas en nuestro país.

\section{¿Qué pasa en Colombia?}

En 1892, Soledad Acosta de Samper (1833-1913) presentó un trabajo titulado "Aptitud de la mujer para ejercer todas las profesiones" en el Congreso Pedagógico Hispano-LusitanoAmericano celebrado en Madrid, en el cual intenta resolver la siguiente pregunta: ¿cuál es la misión de la mujer en la época actual? Afirma a este respecto la connotada escritora:

"Se trata aquí de averiguar si la mujer es capaz de recibir una educación intelectual al igual del hombre, y si sería conveniente darla (sic) suficiente libertad para que pueda (si posee los talentos necesarios) recibir una educación profesional."

Para sustentar la tesis de que la mujer tiene las mismas capacidades que el hombre, da ejemplos del desempeño de las mujeres en diversos campos. Dedica una parte de la segunda sección de su memoria a las mujeres dedicadas a las ciencias: las matemáticas, la astronomía, la botánica, la zoología, la geología y la arqueología, y dice, "Todas estas damas no son aficionadas no más a estudios serios, sino profesoras cuya opinión es acatada por sabios." Termina su escrito afirmando que en el siglo que "empezará en breve" la mujer tendrá la libertad para escoger entre los "recintos científicos que hasta el día de hoy solo frecuentaban los hombres" y "las prerrogativas que en premio de su sumisión y humildad habían gozado en el mundo civilizado desde la Edad Media".

Es durante la República Liberal que en nuestro país se da acceso a la mujer a los estudios de bachillerato. Este paso abrió las puertas para su acceso a la universidad. En el estudio de Cohen, "Mujeres a la vanguardia" (2001), se incluyen entrevistas a las primeras mujeres colombianas que tuvieron acceso al bachillerato y a la universidad. La autora señala que:

"Hacia mediados de la década del sesenta el número de mujeres graduadas en las universidades colombianas había aumentado apreciablemente y en forma continua: de seis que obtuvieron sus títulos en 1938, a 232 en 1955 y a 915 en 1965".

Las mujeres colombianas comenzamos a entrar masivamente a la universidad apenas en los años sesenta del siglo $\mathrm{XX}$; irrumpimos en las aulas de todas las carreras de la Universidad Nacional. La presencia femenina, así fuera escasa, se notaba incluso en las carreras más tradicionales y habitualmente masculinas como la ingeniería, la medicina o el derecho. En esa década se fundó la actual Facultad de Ciencias de la Universidad Nacional que, gracias a la Reforma Patiño, reunía en una sola entidad las distintas carreras de ciencias básicas con orígenes diversos en la Universidad. Las mujeres teníamos una presencia significativa en las carreras de la Facultad: biología, estadística, farmacia, física, geología, matemáticas y química. En el siguiente cuadro se presenta la forma en que las mujeres ingresaron a las carreras de ciencias simultáneamente con los hombres, lo cual se refleja en la fecha de los primeros graduados (Sánchez, 2004).

\begin{tabular}{lccc}
\hline Programa & $\begin{array}{c}\text { Año de } \\
\text { fundación }\end{array}$ & $\begin{array}{c}\text { Año del primer } \\
\text { graduado }\end{array}$ & $\begin{array}{c}\text { Año de la primera } \\
\text { graduada }\end{array}$ \\
\hline Química & 1939 & 1943 & 1944 \\
\hline Matemáticas & 1951 & 1951 & 1966 \\
Geología & 1956 & 1959 & 1960 \\
\hline Física & 1961 & 1966 & 1970 \\
Estadística & 1958 & 1966 & 1968 \\
\hline Biología & 1966 & 1967 & 1968 \\
\hline
\end{tabular}

La carrera de matemáticas en Colombia se fundó en 1951 como Licenciatura en Matemáticas Superiores. Para ingresar al programa se requería haber cursado y aprobado todas las asignaturas de matemáticas de la carrera de ingeniería. El programa continuaba con cursos avanzados en álgebra, análisis y topología. El título de licenciado en ciencias matemáticas se le otorgaba a quienes hubieran aprobado todas las asignaturas y presentado una tesis. Al año siguiente de su apertura, la especialización se convirtió en una carrera profesional de cinco años, a la cual se podía acceder con el título de bachiller. Con este título se graduaron entre 1951 y 1959 seis matemáticos: Pablo Casas Santofimio (1951), Luciano Mora (1953), Erwin von del Walde (1955), José Ignacio Nieto (1956), Guillermo Restrepo (1959) y Alberto Campos (1959).

Con la fundación de esta licenciatura, gracias al entusiasmo de Julio Carrizosa Valenzuela (1895-1974), fundador de la primera Facultad de Ciencias en 1946 bajo la rectoría 
de Gerardo Molina, el italiano Carlo Federici (1906-2005), vinculado desde 1948 a esa facultad, y Pablo Casas (19271983), comenzaron lo que Takahashi (1990) ha dado en llamar "la década dorada de las matemáticas", ya que en 1952 se fundó la primera revista especializada en matemáticas en el país, la Revista de Matemáticas Elementales, por iniciativa del matemático húngaro Juan Horvath (19242015), vinculado como profesor a la Universidad de Los Andes. En 1955 se funda la Sociedad Colombiana de Matemáticas y en 1956 el Departamento de Matemáticas y Estadística de la Universidad Nacional, el cual se convirtió en Facultad en 1958. En 1960 tres jóvenes estudiantes de matemáticas e ingeniería, Jaime Lesmes y los hermanos Carlos y Germán Lemoine, consideraron que su título debía ser el de matemático, ya que en Colombia las licenciaturas están asociadas con la educación básica y media y en la carrera no se consideraban asignaturas de pedagogía ni de didáctica de las matemáticas. Esos tres jóvenes obtuvieron sus títulos de matemáticos y de ingenieros en 1961.

A finales de 1960 comenzó a expresarse en otras universidades colombianas la inquietud por formar matemáticos y licenciados en matemáticas bajo la influencia directa de los primeros egresados de la Universidad Nacional. La primera en hacerlo fue la Universidad de Los Andes, en 1964, seguida por la Universidad del Valle, que creó su programa en 1967, por la Universidad de Antioquia, cuya carrera de matemáticas se inició en 1969 , y por la Universidad Javeriana en 1970.

\section{Colombianas pioneras en matemáticas}

"Las mujeres y las matemáticas, una dupla para destacar" es la tesis de pregrado de Romero-Álvarez (2008) en la Universidad Pedagógica Nacional. Este es un estudio serio que aborda el problema de las matemáticas y el género, y que registra 97 reconocidas matemáticas en diferentes áreas de la disciplina. Romero-Álvarez hizo un sondeo con algunos reconocidos profesores de matemáticas de la Universidad Pedagógica y de la Universidad Nacional para indagar sobre las matemáticas más reconocidas en nuestro país, y los siguientes fueron los nombres de las más destacadas: Myriam Muñoz de Osak, Sonia Sabogal, Tatiana Toro, Clara Helena Sánchez Botero, Mary Falk de Losada, Débora Tejada, Myriam Margarita Acevedo, Doris Hinestroza Gutiérrez, Olga Lucia León, Myriam Ortiz, Lucymar Nova y Clara Marina Neira, todas profesoras de larga trayectoria en Colombia, salvo Tatiana Toro, radicada en los Estados Unidos hace ya varios años. Entre estas escogió a tres, las más "votadas", para hacerles una entrevista: María de Losada, Doris Hinestroza y Clara H. Sánchez. Esta tesis constituye, sin duda, un reconocimiento a las mujeres dedicadas a las matemáticas tanto en Colombia como en el exterior. Varias de ellas son, justamente, las protagonistas de este trabajo.

La presentación que hago de las colombianas pioneras en matemáticas, particularmente de las primeras que obtuvieron un doctorado, se basa en sus propios relatos, y por eso no tienen una estructura uniforme. Es de anotar que el primer título de doctor en Colombia se otorgó en la Universidad Nacional a la química Margot Suárez bajo la dirección de la profesora Carmenza Duque. El primer doctorado en matemáticas se otorgó a José Francisco Caicedo en 1996, y el tercero a Margarita Ospina en el 2000, con lo cual se convirtió en la primera mujer en obtener el doctorado en matemáticas en Colombia. Así pues, las pioneras obtuvieron su doctorado por iniciativa propia en el exterior, ya que en el país todavía no había estudios de doctorado y las universidades no lo exigían para ingresar en ellas como docentes.

Dos hermanas, Clara y Fabiola Rodríguez, fueron las primeras colombianas en obtener el título de "Matemático" en Colombia. Clara lo obtuvo en 1966 (Acta No. 15) y Fabiola en 1970 (Acta No. 29). Ambas se vincularon después de su graduación al Departamento de Matemáticas de la Universidad Nacional, donde permanecieron hasta su retiro. Clara hizo una maestría en matemáticas en la Universidad de Tulane y fue gran colaboradora del profesor Yu Takeuchi (1926-2004) en la edición de la revista Matemáticas Enseñanza Universitaria. Fue la primera en hacer un curso de probabilidad para la carrera de estadística y la primera en publicar un estudio sobre la historia de las mujeres matemáticas (Rodríguez de Takahashi, 1977), planteándose las siguientes preguntas: ¿cuántas mujeres han dejado su nombre en la historia de las matemáticas?, ¿cómo lo lograron y qué hicieron?, ¿se discrimina hoy a la mujer que trabaja en matemáticas? A la primera de estas preguntas respondió reseñando la historia de las pioneras en matemáticas que he mencionado antes, especialmente la de Emmy Noether. Frente a la siguiente pregunta, aunque reconociendo que hoy hay posibilidades para que la mujer se desempeñe en matemáticas, no deja de señalar los prejuicios que subsisten sobre su desempeño.

Nacida en Ibagué, María Margarita Botero de Meza ingresó a la Universidad de Los Andes a la carrera de ingeniería eléctrica. Allí el profesor Henry Yerly (19011984) “empezó a reclutar estudiantes interesados en las matemáticas para que tomaran cursos avanzados de la carrera que estaban planeando abrir. Yo fui una de esos, junto con varios de mis compañeros de ingeniería". Después de graduarse en 1967, se vinculó a la Universidad de Los Andes como profesora y al año viajó a Alemania con una beca y allí obtuvo un Diploma en matemáticas. Regresó en 1970 a Los Andes, pero en 1974 renovaron su beca y volvió a Alemania a continuar el doctorado con su marido y su hija de dos años. Obtuvo el título de Doktor Der Naturwissenschaften de la Technische Universität de Clausthal en 1975. Regresó a la Universidad de Los Andes y desde entonces se ha dedicado a trabajar por las matemáticas y la educación en el país. El decano de la Facultad de Artes y Ciencias de Los Andes le ofreció la jefatura del Departamento de Matemáticas, la cual aceptó, pues, según sus propias palabras, era un reto al que "no me pude negar". Fue directora del Departamento de Matemáticas y gestora de la división de Ciencias y Artes 
en las facultades de Ciencias Naturales y Matemáticas y de Ciencias Sociales y Filosofía durante el periodo de 1984 a 1993. Fue la primera decana de la Facultad de Ciencias. Pasó a dirigir "Una Empresa Docente", centro de educación matemática fundado en Los Andes por Pedro Gómez y luego volvió al Departamento de Matemáticas donde permanece vinculada como profesora emérita desde el 2000. Su mayor orgullo es haber participado en numerosos proyectos interesantes en el área de la educación a través de cursos de capacitación para maestros, talleres y congresos en el país y en el exterior. En este sentido, la maestra dice: "Recuerdo en particular el GTM, Grupo de Trabajo en Matemáticas, y el Comité para el mejoramiento de la enseñanza y la investigación en ciencias básicas, donde junto con colegas de la Nacional, el Valle y la Universidad de Antioquia como Alonso Takahashi y Andrés de la Torre, entre otros, tratamos de hacer un plan de desarrollo de las matemáticas en Colombia, gestionamos préstamos del BID, tratamos de apoyar las colecciones de revistas de matemáticas y mil quijotadas más". Resalta de manera especial el haber participado hasta hoy en algunos proyectos cuyo impactó consideró muy importante: su labor como vicepresidenta de la Asociación Colombiana para el Avance de la Ciencia (ACAC), su gestión en Expociencias y, especialmente, en Expociencia juvenil y Maloka, así como su participación en el inicio y desarrollo de Colfuturo como miembro del comité académico desde su fundación, y en la Asociación Alianza Educativa, AAE, proyecto conjunto de Los Andes y los colegios San Carlos, Nogales y Nueva Granada de Bogotá, mediante el cual administra cinco escuelas en concesión donde estudian más de seis mil niños. Actualmente dirige un proyecto de Los Andes y el Ministerio de Educación cuyo objetivo es crear una red curricular para las matemáticas en los grados primero a once que será publicada en forma de hipertexto y que está sustentada en las reformas curriculares recientemente adoptadas en el mundo, el cual le servirá a los docentes para la construcción y el desarrollo de su plan de aula. La doctora Botero afirma categóricamente: “¡Nunca me sentí ni me he sentido menos, ni por mujer, ni por latina, ni por haber estudiado en el trópico, ni por nada!"

Shirley Bromberg se graduó en 1971 en la Universidad Nacional y se vinculó como profesora al Departamento de Matemáticas. Con una comisión de estudios viajó a México donde obtuvo su maestría y luego su doctorado en el Departamento de Matemáticas del Centro de Investigación y de Estudios Avanzados del Instituto Politécnico Nacional (Cinvestav) bajo la dirección de Juan José Rivaud, con quien también escribió un libro sobre análisis diferencial. Es profesora de la Universidad Autónoma Metropolitana (UAM) desde 1989. Ha tenido una destacada participación en la labor docente en la licenciatura y la maestría de la UAM a través de sus cursos y en la elaboración de textos y materiales didácticos y programas y en el trabajo de coordinación. También ha adelantado una amplia labor de difusión de las matemáticas a través de artículos y conferencias, y de su labor editorial. Su trabajo de investigación abarca tres áreas: la teoría de singularidades, la geometría semiriemanniana y los sistemas dinámicos, sobre los cuales ha escrito diez artículos y dos libros y ha impartido cursos y conferencias en congresos internacionales en Colombia, Guatemala, Nicaragua, Estados Unidos, Alemania, España, Polonia, Uzbekistán y Brasil, así como en numerosos congresos nacionales. También ha realizado tres estancias de investigación en la Universidad de Montpellier, Francia. He incluido algunas de sus publicaciones en la bibliografía (Bromberg, 1976, 2000, 2004, 2007a, 2007b, 2008).

Myriam Muñoz, por su parte, se graduó como matemática en 1971 en la Universidad Nacional. Con una beca del Servicio Alemán de Intercambio Académico (DAAD) viajó a Alemania para estudiar allí después de ser una de las diez personas seleccionadas entre 3.000 aspirantes de todo el mundo. Entre las diez seleccionadas finales había otra colombiana, Laura Martignon. En Alemania obtuvo los siguientes títulos: el Diplom Mathematiker de la Universidad Johannes Gutenberg de Maguncia en 1978 y el Doktor der Naturwissenschaften Nach Mathematik de la Universidad Ruhr de Bochum en 1996, en la cual se le entregó la distinción Magna Cum Laude. Estando allá conoció a su marido turco y se fue a vivir a Turquía. Regresó a Colombia y aprobó el concurso para docentes de la Universidad Nacional. Sus áreas de interés son el análisis y el análisis estocástico. Fue presidenta de la Sociedad Colombiana de Matemáticas entre 1988 y 1990, única mujer que ha ocupado el cargo desde su fundación en 1955, y durante su periodo se destaca el aumento significativo del número de socios. Perteneció al Consejo Directivo Nacional de la ACAC entre 1988 y 1990 y fue segunda vicepresidenta de la Asociación Latinoamericana de Matemáticas entre 1990 y 1994. Durante su vinculación a la Universidad Nacional dirigió siete trabajos de grado en matemáticas, dos de especialización y dos de maestría. Ha participado en congresos, simposios y coloquios a nivel nacional e internacional y tiene 30 publicaciones, la mayoría de ellas en revistas internacionales, algunas de las cuales menciono en la bibliografía (Muñoz, 1992, 1996, 1997, 1998, 2002, 2003). Recibió la distinción de Docencia Excepcional de la Universidad Nacional de Colombia el 17 de diciembre de 2002. Actualmente está "felizmente pensionada", vive en Villa de Leiva y se dedica a hacer artesanías de todo tipo, teniendo muy presente en todo lo que hace su formación matemática.

Después de su grado en Colombia en 1971, Laura Martignon viajó becada a Alemania, como ya lo anoté. Allí obtuvo en 1975 su Diploma en Matemáticas en la Universidad de Tübingen y en 1978 el Doctorado en Ciencias en la misma universidad. Entre 1982 y 1983 fue profesora visitante del Departamento de Matemáticas de la Universidad Hebrea en Jerusalén. En 1979 se vinculó a la Universidad de Brasilia donde fue nombrada como Professor emquadrado com Livre Docencia. En ese país permaneció hasta 1990 ocupando diversos cargos. Entre 1991 y 1993 fue asistente de 
investigación en el Departamento de Neuroinformática de la Universidad de Ulm, y allí obtuvo una beca de investigación (DFG Grant, German Research Community) que se extendió hasta 1995. Ese año se vinculó como investigadora al Centro de Conducta Adaptativa y Cognición (Centro ABC) de la Sociedad Max Planck en Múnich, donde permaneció hasta el 2003. En 1998 obtuvo una habilitación en neuroinformática en la Universidad de Ulm. Desde el 2003 es profesora de matemáticas y de educación matemática con énfasis en diferencias de género en la Universidad Pedagógica de Ludwigsburg. Sus conocimientos en educación matemática y en modelamiento matemático le han permitido trabajar en equipos interdisciplinarios en física y neurociencias. Sus principales contribuciones se han dado en los campos del razonamiento probabilístico y la toma de decisiones. Con Stefan Krauss y otros colegas del Centro ABC y del Instituto de Matemáticas de la Universidad de Ludwigsburg ha propagado los principios de este Centro en torno a las ventajas de los formatos de información natural y la heurística del razonamiento como temas de la educación matemática en el campo de la educación probabilística. Con varios miembros del Harding Center está propagando la alfabetización de riesgo entre niños y jóvenes. Después de haber analizado el razonamiento probabilístico durante décadas, hoy también colabora con Keith Stenning en sus estudios sobre el juicio libre de probabilidades basado en lógicas derogables y su impacto en la educación matemática y en la relación entre género y educación matemática en torno al cual dirige un proyecto en su Universidad y ha fundado la publicación Mathematik und Gender (https://en.wikipedia.org/wiki/ Laura_Martignon). En la bibliografía destaco algunas de sus publicaciones más recientes (Martignon, 1999, 2008a, 2008b, 2009). Debe señalarse, además, que ha obtenido las siguientes patentes: Method for the detection of temporal patterns in the activity of spiking neurons, L. Martignon-G. Deco, German Patent Office (DE 19701931 C1, MPG \& Siemens); Redundancy detector: Method for the detection of redundancy in the activity of spiking neurons, G. Deco -L. Martignon, German Patent Office (DE 19701931 C1, MPG \& Siemens), y Correlator: A user-friendly software for detecting neural assemblies, L. Martignon, G. Deco, 1996, German Patent Office.

Aleida Espinosa, por su parte, es la primera mujer graduada en matemáticas en la Universidad del Valle, título que obtuvo en 1973. Realizó estudios de maestría y fue docente a la Universidad del Valle hasta su retiro.

Nora Donaldson, nacida como Ana Nora Hurtado, debe su apellido a su matrimonio con Simon Donaldson, matemático inglés que recibiera la Medalla Fields en 1982. Obtuvo su título en matemáticas de la Universidad del Valle en 1979 y en 1982 el de maestría en estadística aplicada de la Universidad del estado de Virginia en Estados Unidos. Hizo sus estudios de doctorado en estadística médica en la Universidad de Maryland, Estados Unidos, donde obtuvo el $\mathrm{PhD}$ en 1992. Fue directora de la Unidad de Investigación y
Desarrollo en Bioestadística en el King's College Hospital entre 1999 y 2005, año en que se vinculó al Instituto Dental en Bioestadística de Londres donde trabaja desde entonces. Sus áreas de interés son la bioestadística en odontología, la planeación y evaluación de ensayos clínicos, el modelamiento estructural, el muestreo y las encuestas. Algunas de sus publicaciones están incluidas en la bibliografía (Donaldson, 2009, 2000b, 2009c, 2009d, 2010, 2011).

Débora Tejada es la primera graduada de la carrera de matemáticas de la sede de Medellín de la Universidad Nacional, en 1976. Aunque ingresó a la carrera de ingeniería, allí fue "reclutada" para estudiar matemáticas. Obtuvo una beca en Montpellier donde hizo el doctorado en matemáticas en la Universidad de Ciencias y Técnicas de Languedoc, y en 1981 obtuvo un doctorado de tercer ciclo. Regresó a Colombia y se vinculó como docente de matemáticas en las Universidades Nacional y de Antioquia. Allí conoció a su marido, Jorge Cossio, y juntos viajaron en comisión a los Estados Unidos para hacer un programa de doctorado. La doctorabTejada se graduó en 1993 con una tesis en topología con especialidad en cubiertas ramificadas en la Universidad del Norte de Texas. Sus áreas de interés son la topología y la teoría de nudos, campos en los que ha publicado, por lo menos, 20 trabajos en revistas nacionales e internacionales o en capítulos de libro. Ha participado en seis proyectos de investigación financiados por Colciencias, y dirigió ocho tesis de maestría, entre ellas las tres primeras producidas en la Maestría en Matemáticas de la sede de Medellín de la Universidad Nacional de Colombia. Ha dado más de 50 conferencias en diversos eventos de carácter nacional e internacional, y en el 2010 fue invitada como ponente al Congreso Internacional de Matemáticas realizado en Hyderabad, India. En la bibliografía destaco varias de sus publicaciones (Tejada, 2001, 2002, 2004, 2005, 2006, 2011). Es profesora emérita de la Universidad Nacional, sede Medellín, desde el 2011, y estuvo vinculada al proyecto "Plan de mejoramiento de la enseñanza de la matemática en las instituciones educativas de Antioquia", durante la gobernación de Sergio Fajardo. Es hija de dos ingenieros, José Tejada Sáenz (1919-2011) y Sonny Jiménez (19222014), y su madre fue la primera mujer colombiana que obtuvo el título de ingeniera civil en Colombia, en 1946, en la Facultad de Minas de la Universidad Nacional. Débora destaca el valor de su familia en su formación.

Doris Hinestroza se graduó como Matemática de la Universidad del Valle en 1977 y fue la primera en obtener la maestría de esa misma Universidad en 1982. Obtuvo una beca y realizó estudios de maestría y doctorado en la Universidad de Cincinnati y obtuvo sus grados en 1990 y 1992, respectivamente. Su área de especialización en matemáticas aplicadas es la de problemas inversos mal puestos. Es Profesora distinguida y Maestra universitaria de la Universidad del Valle donde ha ocupado varios cargos, entre otros, el de Directora del Departamento de Matemáticas, Decana de la Facultad de Ciencias y Directora del Posgrado. 
Las dificultades económicas de su infancia y juventud no fueron obstáculo para lograr sus objetivos. Desde pequeña percibió que le gustaban las matemáticas y ser "maestra". Así que, aunque es investigadora destacada en sus temas de interés, valora de manera especial su ejercicio como docente. Lidera la Asociación Colombiana de Mujeres Matemáticas, consciente de que es necesario trabajar por la comunidad matemática colombiana y particularmente por el estímulo a las niñas y jóvenes para que se formen en esta disciplina. En la bibliografía se destacan varias de sus publicaciones (Hinestroza, 2002, 2004, 2013, 2013b, 2013c, 2017).

Margarita Ospina Pulido afirma que, "Desde el inicio de la carrera en Matemáticas en 1979, donde éramos nueve o diez mujeres entre 80 admitidos, se hizo presente la constancia y la tenacidad femenina para culminar con éxito los estudios. Los graduados de esa cohorte fuimos alrededor de unos diez y entre ellos tres mujeres. En la maestría, aunque no recuerdo bien, las proporciones de hombres a mujeres estaban más equilibradas, así como su índice de graduación. $\mathrm{Al}$ abrirse el primer programa de doctorado en Colombia en la Universidad Nacional, sede Bogotá, eran muchas las expectativas sobre los primeros admitidos y los primeros graduados. Fuimos admitidos 11 aspirantes y entre ellos tres mujeres: Clara Neira, Sonia Sabogal y yo. A decir verdad, me sorprendió ser admitida, porque aunque mis calidades académicas las había demostrado en la carrera y la maestría, en ese momento era una madre de familia con dos hijos de 3 y 4 años que había dejado en receso sus actividades académicas durante cuatro años. Sonia, otra de las candidatas, también era madre de dos hijos de aproximadamente 3 y 10 años. De esa cohorte nos graduamos cinco personas; dos hombres y las tres mujeres. Las tres trabajamos arduamente con unos directores de tesis reconocidos por sus niveles de exigencia: los doctores Januario Varela y Carlos Ruiz. Nunca sentí discriminación ni a favor ni en contra. Colciencias y la Fundación Mazda también creyeron en nosotras, pues Sonia y yo fuimos becarias de las dos entidades. También destaco que mis logros no son solo míos, hacer un doctorado en las condiciones que yo lo hice requiere de un esposo y padre de mis hijos excepcional: mi colega y esposo Lorenzo Acosta". Margarita obtuvo su título de pregrado en 1984, durante un tiempo trabajó como docente en varias universidades en Bogotá: la Universidad Distrital, la Universidad de los Andes y la Universidad Javeriana. En 1994 ingresó al Departamento de Matemáticas de la Universidad Nacional en Bogotá, al cual sigue vinculada. Sus áreas de interés son la topología, la teoría de categorías, y la enseñanza y la evaluación en matemáticas. Desde hace unos años se dedica especialmente a estudiar el impacto, la evolución y el mejoramiento de los cursos de nivelación en matemáticas básicas de la Universidad Nacional, sede Bogotá. Fue constructora y evaluadora de preguntas abiertas de matemáticas en las pruebas del Examen de estado de calidad de la educación superior entre 2005 y 2009 . Actualmente es miembro del equipo de constructores de las pruebas de admisión de la Universidad
Nacional y del equipo de construcción de las pruebas de eficiencias en matemáticas de la Facultad de Ciencias de esa universidad. Por sus calidades docentes recibió la distinción de Docencia meritoria de la Universidad Nacional, sede Bogotá, en el 2013. En la bibliografía se destacan algunas de sus publicaciones (Ospina, 1999, 2000, 2004, 2016).

\section{Conclusiones}

Las mujeres colombianas del siglo XX no solo llegaron a los recintos de la ciencia sino que rechazaron aquellas prerrogativas que tanto valoraba doña Soledad. El ingreso de la mujer colombiana a la universidad se da en los años 30 , con la llegada de los liberales al poder y la reforma de la educación de López Pumarejo. La Universidad Nacional se reorganizó mediante la Ley 68 de 1935, lo cual también fue un factor decisivo para el cambio. Una de las primeras en aprovechar esta oportunidad fue Sonny Jiménez de Tejada, quien, como ya anoté, fue la primera graduada en ingeniería civil en la Escuela de Minas.

Creo que las mujeres somos más persistentes para lograr nuestros objetivos. Creo que asumimos los múltiples papeles que debemos desempeñar como esposas, madres, hijas, amantes, novias, amigas, sin descuidar ese otro rol como profesionales responsables y eficientes. Pero creer no basta en la ciencia, hay que demostrar con estudios serios que así es. Por ello es esperanzador que en Colombia comiencen a desarrollarse los estudios de género en la ciencia y en las matemáticas (Rojas \& Correa, 2014).

\section{Conflicto de intereses}

La autora declara no tener conflicto de intereses.

\section{Referencias}

Acosta de Samper, S. (1892) Aptitud de la mujer para ejercer todas las profesiones. Memoria presentada en el Congreso Pedagógico Hispano-Lusitano-Americano celebrado en Madrid.

Bell, E. T. (1937) Men of Mathematics. Simon and Schuster Editors.

Boisseau, A., Chauveau, V., Delon F., Modec, G., (2000) Du côté des mathématiciennes. ALÉAS. Lyon.

Bromberg S., Rivaud,J.J (1976) Análisis diferencial. Fondo de Cultura Económica, México.

Bromberg, S., Medina A. (2008) Geodesically complete Lorentzian metrics on some homogeneous 3 manifolds. Symmetry, Integrability and Geometry: Methods and Applications. 4: 1-13.

Bromberg, S., López de Medrano, S. (2007a) Differentiability and composite function. Singularity Theory. World Scientific Press. 463-474.

Bromberg, S., Medina A. (2007b) A remark on the completeness of homogeneous quadratic vector fields on the plane. Qualitative Theory of Dynamical Systems. 6 (2): 181-186.

Bromberg, S., Medina A. (2004) Geometry of oscillator groups and locally symmetric manifolds. Geometriae Dedicata. 106: $97-111$.

Bromberg, S. (2000) A homogeneous space-time model with singularities. J. Math. Phys. 41 (12): 8190-8195. 
Byers, N., Williams, G. (2010). Out of the shadows. Contributions of twentieth-century women to physics. Cambridge University Press. Boston, 471p.

Clarke E.H. (1884). Sex in Education or A fair chance for girls. The Riverside Press, Cambridge. 471p.

Cohen, L. (2001). Colombianas en la Vanguardia. Editorial de la Universidad de Antioquia. Medellín. 351p.

Donaldson, N. (2009). Widening access? Characteristics of applicants to medical and dental schools, compared with UCAS. British Dental Journal. 207 (9): 433-445.

Donaldson, N. (2009b). Use of the out-of-hours emergency dental service at two south-east London hospitals. Bmc Oral Health. 10 (1186): 9-19.

Donaldson, N. (2011). Systematic review and assessment of oral health promotion programs in the UK. European Journal of Dental Education: Official Journal Of The Association For Dental Education. 15: 1-8.

Donaldson, N. (2010). The Effects of Dental Anxiety and Irregular Attendance On Referral For Dental Treatment Under Sedation Within The National Health Service In London. Community Dentistry and Oral Epidemiology. 38 (5): 453-459.

Donaldson, N. (2009c). Dental age assessment (DAA): Reference data for British Caucasians at the 16-year threshold. Forensic Science International. 189 (1-3): 19-23.

Donaldson, N. (2009d). Reliability and cross-cultural validity of a Japanese version of the Dental Fear Survey. Bmc Oral Health. 10 (1186): 9-17.

Dieudonné, J. (1989). En honor del espíritu humano. Alianza Editorial. Madrid.

Ernest, J. (1976). Mathematics and sex. The American Mathematical Monthly. 83 (8): 595-614.

Ernest, J. (2000). Los valores y la imagen de las matemáticas: una perspectiva filosófica. Matemáticas, cultura y sociedad. Uno. Revista de didáctica de las matemáticas. p. 1-16.

Halpern, D.F., Benbow, C. P., Geary, D.C. Geary, G., Ruben, C., Hyde, J. S., Gernsbacher, M. A. (2007). La ciencia de las diferencias sexuales en ciencia y matemáticas. Psychological Science for the Public Interest. Association for Psychological Science. 8 (1): 1-51.

Henrion, C. (1997). Women in mathematics, Indiana, USA: University Press. xxxi+293p.

Hinestroza, D., Ganio, C., A Fraguela, A. (2002). The inverse problem of electrical capacitance and its application to gasoil. Wseas Transactions on Systems. 1 (2): 130.

Hinestroza, D., Ganio, C. (2004). Regularization techniques for non-linear problems applied to electrical capacitance tomography. Aportaciones Matematicas. Parametric Optimization and Related Topics VII. 18: 143-153.

Hinestroza, D., Aponte Mayor, G., Pleite Guerra, J., Silva, D.F. (2013). Obtaining the electrical impedance using wavelet transform from the time response. Ieee Transactions on Power Delivery. 28 (2): 1242-1244.

Hinestroza, D., Aponte Mayor, G., Pleite Guerra, J., Silva, D.F. (2013b). Application of wavelet transform to obtain the frequency response of a transformer from transient signals. Part 1: Theoretical analysis. Ieee Transactions on Power Delivery. 28 (2): 1709-1714.

Hinestroza, D. Olivar, L.E., Peralta Navarro, J. (2013c). Regularization algorithm within two parameters for the identification of the heat conduction coefficient in the parabolic equation. Mathematical and Computer Modelling. 57 (7-8): 1990-1998.
Hinestroza, D. (2017). Coefficient identification in the EulerBernoulli equation using regularization methods. Applied Mathematical Modelling. 41: 223-235.

Martignon L., Katsikopoulos, K.V., Woike, J.K. (2008). Categorization with limited resources: A family of simple heuristics. Journal of Mathematical Psychology.

Martignon, L., Krauss, S. (2009). Hands-on activities for fourth graders: A tool box for decision-making and reckoning with risk. Mathematics Education. p. 227-258.

Martignon L., de Haan, G., Kamp, G., Lerch, A., MüllerChrist, G., Nutzinger, H.G. (2008). Nachhaltigkeit und Gerechtigkeit: Grundlagen und schulische Konsequenzen, Springer: New York.

Martignon L., Gigerenzer, T., ABC Group. (1999). Simple heuristics that make us smart. Oxford University Press.

Muñoz M. (1992). Discrete time Markov Control Process with discounted unbounded costs: Optimality criteria. Kybernetika. 28: 191-212.

Muñoz M. (1996). Nonstandard Construction of Brownian motion and Martingales on Lie Groups. Dissertations Sum-maries in Mathematics. 1: 259-266.

Muñoz M. (1997). Quadratic variation and integration in a two parameter stochastic analysis means nonstandard analysis. Proceedings of the 51th. Session of the Interna-tional Statistical Institute. Istanbul. p. 549-550.

Muñoz M. (1998). Lifting theorems for some classes of two parameter martingales. Revista Colombiana de Matemáticas. 32 (1): 45-70.

Muñoz M., Blanco, L. (2002). Introducción a la teoría avanzada de la Probabilidad. Universidad Nacional de Colombia, Bogotá.

Muñoz M., Blanco, L. (2003). Análisis estocástico. Notas de clase. Facultad de Ciencias. Universidad Nacional, Bogotá.

Osen, L. M. (1974). Women in Mathematics. Cambridge, Massachusetts, and London: The MIT Press.

Ospina, M., Acosta L, Bateman J, Monsalve S, Isaacs R, Ruiz C. (1987). Una aproximación booleana a la topología general. IV Coloquio Distrital de Matemáticas y Estadística, Bogotá.

Ospina, M. (1999). Estructuras de O-categoría generadas por funtores fieles y epi-objetos. Boletín de Matemáticas. Nueva Serie. VI (1): p. 69-75.

Ospina, M. (2000). Una introducción a las O-categorías. Cursillo XVII Coloquio Distrital de Matemáticas y Estadística, Bogotá.

Ospina, M., Buitrago, J. (2004). Sobre el compactado de WallmanFrink. Memorias del XXI Coloquio Distrital de Matemáticas.

Ospina, M. (2016). Curso matemáticas básicas para ciencias, ciencias económicas e ingenierías. Colección Notas de clase, Facultad de Ciencias, Universidad Nacional de Colombia, Bogotá, Editorial UN. Primera reimpresión 2017.

Paycha, S., Georgescu, M., Azzali, S. (2016). Women of mathematics throughout Europe. A gallery of portraits. Berlin: Verlag am Fluss. 122p.

Perdomo, I. (s.f.) Matemáticas y género. Una aproximación histórica. Accesible en: www.oei.es/historico/salactsi/Matematicas yGeneroUnaaproximacionhistorica1.pdf

Rodríguez de Takahashi, C. (1977). Las matemáticas. Matemática Enseñanza Universitaria. p. 31-41.

Rojas Betancourt, M., Correa Álvarez, D. (2014). ¿El género en matemáticas? Un análisis de los resultados de las olimpiadas matemáticas. Escenarios. 12 (1): 7-16. Accesible en: repositorio.uac.edu.co/handle/11619/1419 
Romero Álvarez A. A. (2008). Las mujeres y las matemáticas, una dupla para destacar. Tesis de grado para optar al título de Licenciado en Matemáticas. Universidad Pedagógica Nacional, Facultad de Ciencia y Tecnología, Departamento de Matemáticas.

Sánchez, C.H. (1988). Mujeres pioneras en matemáticas. Notas de Matemática. 25: 47-66.

Sánchez, C.H. (2004). Mujeres en la Facultad de Ciencias de la Universidad Nacional. Memorias del seminario Las mujeres colombianas en el sistema de ciencia y tecnología: obstáculos y logros. ICANH, Bogotá.

Sánchez C.H. (2007a). Los ingeniero-matemáticos colombianos del siglo XIX y comienzos del XX. Universidad Nacional, Facultad de Ciencias, Bogotá.

Sánchez C.H. (1995). Soluciones no planas a los famosos problemas de construcción. Cuadernillo. XI Coloquio Distrital de matemáticas y estadística.

Sánchez C.H. (1996). El Surgimiento de la teoría de conjuntos. Cuadernillo, Coloquio Distrital de Matemáticas y Estadística, Bogotá.

Sánchez C.H. (1997). La construcción de los números reales. Cuadernillo. XIV Coloquio Distrital de Matemáticas y Estadística, Bogotá.

Sánchez C.H. (1985). Antecedentes trigonométricos de la teoría de conjuntos. Lecturas Matemáticas. 5: 61-78. Cadernos de Historia e Filosofia da Ciencia. 10: 61-69.

Sánchez C.H., Albis V. (1999). Descripción del curso de cálculo diferencial de Aimé Bergeron en el Colegio Militar. Revista de la Academia de Ciencias Exactas, Físicas y Naturales. 23 (86): 73-79.

Sánchez C.H. (1999). Matemáticas en Colombia en el siglo XIX. Llull. 22: 687-705.

Sánchez C.H. (2002). Cien años de historia de la matemática en Colombia 1848-1948. Revista de la Academia Colombiana de Ciencias Exactas, Físicas y Naturales. 26 (99): 239-260.

Sánchez C.H. (2007b). Los cuadernos de Julio Garavito. Una antología comentada. Revista de la Academia Colombiana de Ciencias Exactas, Físicas y Naturales. 31: 253-266.

Sánchez C.H. (2007c). Contribuciones a la teoría de números transfinitos. Una introducción. Mathesis. III2 2 : 345-385.

Sánchez C.H. (2009). La introducción de la teoría de conjuntos y la matemática moderna en Colombia. Primera parte: el aporte de los extranjeros. Mathesis. III4 2 : 265-293.
Sánchez C.H. (2012). La historia como recurso didáctico: el caso de los Elementos de Euclides. Tecne, Episteme y Didaxis. 32: 71-92.

Sánchez C.H. (2012). Historia de la enseñanza de las matemáticas en Colombia. De Mutis al siglo XXI. Quipu. 14: 109-157.

Santos, D., Ursini, S., Ramírez, M., Sánchez, G. (2006) Mathematics achievements: Sex differences Vs. gender differences. In Novotná, J., Moraová, H., Krátká, M. \& Stehlíková, N. (Eds.). Proceedings 30th Conference of the International Group for the Psychology of Mathematics Education. 5: 41-48.

Takahashi, A. (1990). Estudios sobre el estadio de desarrollo y de inserción social de las disciplinas y áreas del conocimiento. Matemáticas. La conformación de sociedades científicas en Colombia. Misión de Ciencia y Tecnología. MEN, DNP, Fonade. 3 (1): 5-205.

Tejada D., Salingaros N. (2001). Modularity and the number of design choices. Nexus Network Journal. Architecture and Mathematics. 3 (1): 99-109.

Tejada D., Toro Villegas, M.M., Mike Hilden, M. (2002). Tunnel number of one knots have palindrome presentations. Journal of Knot Theory and Its Ramifications. 11 (5): 815-831.

Tejada D., Toro Villegas, M.M. Hilden, M., Montesinos J. J. (2004). Mariposas y 3-variedades. Revista de la Academia Colombiana de Ciencias Exactas, Físicas y Naturales. 28 (106): 71-78.

Tejada D., Toro Villegas, M.M. Hilden, M., Montesinos J.J (2005). Representing 3-manifolds by triangulations of $\mathrm{S}^{3}$ : A constructive approach. Revista Colombiana de Matemáticas. 39 (2): 63-86.

Tejada D., Toro Villegas, M.M. Hilden, M., Montesinos J.J. (2006). Fox coloured knots and triangulations of $\mathrm{S}^{3}$. Mathematical Proceedings of The Cambridge Philosophical Society. 141 (3): 443-463.

Tejada D., Hilden, M., Montesinos J.J. (2011). Artifacts for stamping symmetric designs. American Mathematical Monthly. 18 (4): 327-343.

Revista National Geografic. (2017). Género. La revolución. El Panorama cambiante del género. Edición especial. National Geograpic, enero 2017. 